\title{
Effect of Earth Albedo Variation on the Performance of a Spatial Acquisition Subsystem Aboard a Planetary Spacecraft
}

\author{
C. - C. Chen \\ Communications Systerns Research Section
}

\begin{abstract}
The effect of Earth albedo variation on the pointing and tracking subsystem of a planetary optical communication package is analyzed. By studying the Cramer-Rao bound of the tracking error variance, it is shown that, when the Earth albedo is precisely known, the variance in spatial tracking error is inversely proportional to the total signal count. In contrast, a small uncertainty in the Earth albedo can result in an irreducible error in the tracking subsystem.
\end{abstract}

\section{Introduction}

Accurate spatial acquisition and tracking are critical for the operation of free-space optical communication systems. In order to maintain the signal power loss to within an acceptable level, tracking accuracy on the order of $1 / 10$ to $1 / 20$ of the transmitted beamwidth is generally required. For a system operating at $0.5-\mu \mathrm{m}$ wavelength using a $30-\mathrm{cm}$ aperture, the desired pointing accuracy will be on the order of $0.1 \mu \mathrm{rad}$. Since the angular resolution of the optical system is roughly equal to its transmitted beamwidth, the narrow pointing requirement implies that the spatial acquisition subsystem must derive a pointing reference to within 10 percent of the minimal spatial resolution. Because both the transmitter and the receiver are typically in motion, the tracking information must be derived within a time period during which the receiver may move a significant distance within the transmitter field-of-view. In some systems, the residual vibration due to the mechanical system will be much larger than the desired pointing accuracy. For these systems, the tracking information must be obtained at a rate that is higher than the vibration frequency so that the optical system can effectively compensate for vibrationinduced error.

The performance of spatial tracking algorithms has been investigated by several authors [1], [2]. Most of these studies, however, were carried out with the assumption that a beacon signal is available as a pointing reference. These studies generally suggest that the effective pointing error decreases with increasing beacon strength or, effectively, with increasing integration time. In the latter case it is assumed that the beacon strength remains constant so that increasing the integration time, i.e., decreasing the tracking loop bandwidth, will result in an effective increase of the detector signal-to-noise ratio (SNR). In some cases, however, an active pointing beacon can be either undesirable or unfeasible. In these cases it may be desirable for the transmitter to use the a priori knowledge of the receiver to derive the pointing information. For instance, a spacecraft may use the sun-lit Earth as a pointing reference 
and derive the actual receiver location using simple geometric rules. This scheme can be particularly attractive for a deepspace optical communication system for which the uplink beacon may require several kilowatts of radiated power.

The problem of deriving a pointing reference is equivalent to locating the image of the object on the receiver focal plane. For an extended object that can be resolved by the telescope, the optimal maximum-likelihood tracking algorithm has been derived, and its performance has been extensively investigated. The results generally state that, given the known source intensity distribution, the variance of tracking error will be inversely proportional to the detector SNR. Unfortunately, for most applications, the source brightness distribution is not precisely known. Consequently, there will be an error associated with the spatial tracking subsystem. The purpose of this report is to analyze the effect of uncertainties in source brightness on the spatial tracking subsystem.

The rest of this paper is organized as follows. Section II describes the conventional maximum-likelihood tracking algorithm for determining the pointing reference. The effect of uncertainties in source brightness will be analyzed in Section III by studying the Cramer-Rao bound on the tracking error variance. A typical planetary optical communication package using the sun-lit Earth as a pointing reference will then be described and the impact of Earth albedo variation on the tracking error will be studied in Section IV. Finally, the results from the study will be summarized in Section V.

\section{Maximum Likelihood Spatial Tracking Algorithm}

In this section the Maximum Likelihood (ML) algorithm for determining the angular position of the receiver is derived. It is assumed that the shape and orientation of the receiver is known. The problem of estimating the angular coordinate of the receiver is equivalent to estimating the location of the receiver image on the receiver focal plane. Without loss of generality, this is equivalent to locating a fixed reference point on the image. For simplicity, the reference point is chosen to be the geometric center $r$ of the image. Consequently, the problem of spatial tracking can be reduced to the problem of estimating the geometric center given the detector photocount statistics and the prior knowledge of the source brightness distribution, $I_{0}(\rho)$, where $\rho$ is the distance to the image center.

The problem of spatial estimating is complicated by the fact that the receiver has a finite spatial resolution. This is because the tracking detector, which is typically a charge-coupled device (CCD), has discrete spatial cells (pixels) that occupy a finite area. Each pixel output represents the total intensity of light impinged on the pixel area. Furthermore, because of the granularity of the optical signal, the output of the $(i, j)^{\text {th }}$ pixel $k_{i j}$ will be a Poisson-distributed random variable with a mean $\lambda_{i j}(r)$ where

$$
\lambda_{i j}(r)=\int_{A_{i j}} I_{0}(\rho-r) d^{2} \rho
$$

In writing Eq. (1), $I_{0}(\rho-r)$ denotes the brightness distribution of the image where the geometric center is displaced by an amount $r$, and the integral is over the surface of the $(i, j)^{t h}$ pixel.

The problem of deriving the transmitter pointing reference is therefore reduced to the problem of estimating the deviation $r$ from a set of detector photocounts, $\left\{k_{i j}\right\}$. The maximum a posteriori (MAP) estimator [3] of the deviation $\widehat{r}_{\text {MAP }}$ is given by

$$
\widehat{r}_{\mathrm{MAP}}=\arg \left\{\max _{r}\left\{P\left(r \mid\left\{k_{i j}\right\}\right)\right\}\right\}
$$

If it is assumed that the prior probability distribution of $r_{0}$ is uniformly distributed, then the decision rule can be reduced to the following maximum-likelihood (ML) rule:

$$
\begin{aligned}
\hat{r}=\arg \left\{\max _{r}\left\{P\left(\left\{k_{i j}\right\} \mid r\right)\right\}\right\}= & \arg \left\{\operatorname { m a x } _ { r } \left\{\log \prod_{i=1}^{N} \prod_{j=1}^{N} \frac{\lambda_{i j}(r)^{k_{i j}}}{k_{i j} !}\right.\right. \\
& \left.\left.\times e^{-\lambda_{i j}(r)}\right\}\right\}
\end{aligned}
$$

where it is assumed that $k_{i j}$ are independent and Poissondistributed with parameter $\lambda_{i j}(r)$, and observed that the logarithmic transformation does not change the maximum of a function. The ML decision rule can be further simplified by realizing that

$$
\sum_{i=1}^{N} \sum_{j=1}^{N} \lambda_{i j}(r)
$$

is proportional to the total power received by the receiver, and is therefore independent of the location of the geometric center $r$. Consequently, the ML decision rule can be written as

$$
\hat{r}=\arg \left\{\max _{r}\left\{\sum_{i=1}^{N} \sum_{i=1}^{N} k_{i j} \log \lambda_{i j}(r)\right\}\right\}
$$

The performance of the estimator can be estimated by using the Cramer-Rao lower bound (CRLB) [1] 
$\operatorname{Var}\left(\left|\hat{r}-r_{0}\right|\right) \geqslant$

$$
\left[\frac{E\left[\left(\frac{\partial F(r)}{\partial x}\right)^{2}+\left(\frac{\partial F(r)}{\partial y}\right)^{2}\right]}{E\left[\left(\frac{\partial F(r)}{\partial x}\right)^{2}\right] E\left[\left(\frac{\partial F(r)}{\partial y}\right)^{2}\right]-\left\{E\left[\frac{\partial F(r)}{\partial x} \frac{\partial F(r)}{\partial y}\right]\right\}^{2}}\right]_{r=r_{0}}
$$

where

$$
F(r)=\sum_{i j} k_{i j} \log \lambda_{i j}(r)
$$

and $E[x]$ denotes the expectation value (over $\left\{k_{i j}\right\}$ ) of the variable $x$. By using the assumption that $k_{i j}$ are independent and Poisson-distributed, the lower bound can be further reduced to

$$
\operatorname{Var}\left(\left|\hat{r}-r_{0}\right|\right) \geqslant\left[\frac{\sum_{i j} \frac{\left(\partial \lambda_{i j}(r) / \partial y\right)^{2}+\left(\partial \lambda_{i j}(r) / \partial x\right)^{2}}{\lambda_{i j}(r)}}{\left[\sum_{i j} \frac{\left(\partial \lambda_{i j}(r) / \partial y\right)^{2}}{\lambda_{i j}(r)}\right]\left[\sum_{i j} \frac{\left(\partial \lambda_{i j}(r) / \partial x\right)^{2}}{\lambda_{i j}(r)}\right]-\left[\sum_{i j} \frac{\left(\partial \lambda_{i j}(r) / \partial x\right)\left(\partial \lambda_{i j}(r) / \partial y\right)}{\lambda_{i j}(r)}\right]^{2}}\right]_{r=r_{0}}
$$

Given the source intensity distribution, $I_{0}(\rho)$, the variance of tracking error can be calculated. It should be noted that the CRLB is a lower bound on the estimator error. However, it provides an analytically tractable expression, and is therefore very useful in estimating the performance of the tracking system.

Note that the variance in Eq. (6) is in general a function of $r_{0}$, the actual geometric center. Furthermore, the variance depends on both the shape of the image as well as its intensity. To study the effect of increasing source intensity, or equivalently, increasing the integration time, one can normalize the receiver count parameter $\left\{\lambda_{i j}(r)\right\}$ as

$$
\lambda_{i j}(r)=N_{0} g_{i j}(r)
$$

where $N_{0}$ is the average number of photons received over the tracking sensor, and $g_{i j}(r)$ is the fraction of photons that falls onto the $(i, j)^{t h}$ pixel. By definition,

$$
\sum_{i j} g_{i j}(r)=1
$$

Given the above definition, the CRLB can be written as

$$
\begin{aligned}
\operatorname{Var}\left(\left|\hat{r}-r_{0}\right|\right) \geqslant \frac{1}{N_{0}}\left[\frac{\sum_{i j} \frac{\left(\partial g_{i j}(r) / \partial y\right)^{2}+\left(\partial g_{i j}(r) / \partial x\right)^{2}}{g_{i j}(r)}}{\left[\sum_{i j} \frac{\left(\partial g_{i j}(r) / \partial y\right)^{2}}{g_{i j}(r)}\right]\left[\sum_{i j} \frac{\left(\partial g_{i j}(r) / \partial x\right)^{2}}{g_{i j}(r)}\right]-\left[\sum_{i j} \frac{\left(\partial g_{i j}(r) / \partial x\right)\left(\partial g_{i j}(r) / \partial y\right)}{g_{i j}(r)}\right]_{r=r_{0}}^{2}}\right] \\
=\frac{1}{N_{0}} G\left(r_{0}\right)
\end{aligned}
$$

It is easily seen that the performance of the tracking system improves with increasing signal power, $N_{0}$. The function $G\left(r_{0}\right)$ in Eq. (8) depends only on the shape of the image, and not its intensity. Figure 1 shows the values of $G\left(r_{0}\right)$ for two very simple cases. For a more general image shape, $G\left(r_{0}\right)$ is very difficult to calculate. However, it can be seen from Eq. (8) that the lower bound in error variance is minimized when 


$$
\begin{aligned}
& {\left[\sum_{i j} \frac{\left(\partial g_{i j}(r) / \partial x\right)^{2}}{g_{i j}(r)}\right]_{r=r_{0}} \gg 1} \\
& {\left[\sum_{i j} \frac{\left(\partial g_{i j}(r) / \partial y\right)^{2}}{g_{i j}(r)}\right]_{r=r_{0}} \gg 1}
\end{aligned}
$$

and

$$
\left\{\left[\sum_{i j} \frac{\left(\partial g_{i j}(r) / \partial x\right)\left(\partial g_{i j}(r) / \partial y\right)}{g_{i j}(r)}\right]_{r=r_{0}}\right\}^{2} \ll\left[\sum_{i j} \frac{\left(\partial g_{i j}(r) / \partial x\right)^{2}}{g_{i j}(r)}\right]_{r=r_{0}}\left[\sum_{i j} \frac{\left(\partial g_{i j}(r) / \partial y\right)^{2}}{g_{i j}(r)}\right]_{r=r_{0}}
$$

The expressions in Eqs. (9a) and (9b) are greater for images with high contrasts, i.e., images that contain pixels with high $\left|\partial g_{i j}(r) / \partial x\right|$ and $\left|\partial g_{i j}(r) / \partial y\right|$. Since the reference image is usually much brighter than the background, the partial derivatives are greater near the image border. It follows that the CRLB is smaller for images with better defined borders, i.e., images for which $\partial g_{i j} / \partial x$ and $\partial g_{i j} / \partial y$ are large.

\section{Albedo Variation}

The derivation above shows that, when the source intensity distribution is known, the variance in estimating the image location decreases linearly with increasing signal power. For a sufficiently bright source, the variance is negligible.

Unfortunately, the derivation that leads to Eq. (8) assumes that the exact source intensity distribution $I_{0}(r)$ is known at the receiver. In some cases, the intensity distribution of the receiver can be quite unpredictable. For example, for a deepspace vehicle using the sun-lit Earth as the pointing reference, the albedo variation of the Earth can be caused by weather patterns and changing surface conditions. Furthermore, these conditions are in general time-varying so that they cannot be expected to remain constant.

In order to quantify the effect of intensity uncertainty on the spatial tracking subsystem, some assumptions on the intensity error distribution are required. For this analysis, it is assumed that the estimated source intensity distribution $\widehat{I}(\rho)$ differs from the actual source intensity $I_{0}(\rho)$ by a small amount $I_{1}(\rho)$. Furthermore, it will be assumed that $I_{1}(\rho)$ is a zero-mean Gaussian random process with an autocorrelation function

$$
\phi\left(\rho, \rho^{\prime}\right) \equiv\left\langle I_{1}(\rho) I_{1}\left(\rho^{\prime}\right)\right\rangle
$$

The maximum likelihood estimator must estimate the location of the image based on an incomplete estimate of the source distribution. In other words, the estimate $\hat{r}$ for the image center can be written as

$$
\widehat{r}=\arg \left[\max _{r} P\left(\left\{k_{i j}\right\} \mid r, \hat{I}(\rho)\right)\right]=\arg \left\{\max _{r} F^{\prime}(r)\right\}
$$

where $F^{\prime}(r)=\log \left[P\left(\left\{k_{i j}\right\} \mid r, \widehat{I}(\rho)\right)\right]$ is the likelihood function, and we have used the fact that a logarithmic transformation does not affect the location of a functional maximum.

Given the formulation of the estimator in Eq. (11), the variance of the estimation error can again be given in terms of its Cramer-Rao lower bound as in [1]

$$
\begin{aligned}
& \operatorname{Var}\left(\left|\hat{r}-r_{0}\right|\right) \geqslant \\
& {\left[\frac{E\left[\left(\frac{\partial F^{\prime}(r)}{\partial x}\right)^{2}+\left(\frac{\partial F^{\prime}(r)}{\partial y}\right)^{2}\right]}{E\left[\left(\frac{\partial F^{\prime}(r)}{\partial x}\right)^{2}\right] E\left[\left(\frac{\partial F^{\prime}(r)}{\partial y}\right)^{2}\right]-\left\{E\left[\frac{\partial F^{\prime}(r)}{\partial x} \frac{\partial F^{\prime}(r)}{\partial y}\right]\right\}^{2}}\right]_{r=r_{0}}}
\end{aligned}
$$

The expectation in Eq. (12) is, in general, very complicated since the detector photocounts $\left\{k_{i j}\right\}$ are conditional Poissondistributed random variables. Given the estimated intensity $\hat{I}(\rho)$, the actual source intensity distribution $I_{0}(\rho)$ can be modeled as a random variable with mean $\hat{I}(\rho)$. That is, the mean photocount expected over the $(i, j)^{t h}$ pixel, $\lambda_{i j}$, is a random variable with mean 


$$
\hat{\lambda}_{i j}(r)=\int_{I_{i j}} \hat{I}(\rho-r) d^{2} r
$$

When the source intensity is sufficiently high, or over a sufficiently long integration time, the fluctuation in the Poisson count statistics will be small compared to its mean. In this case one can approximate the detector photocount in Eq. (11) by its mean value, and the likelihood function in Eq. (11) can be reduced to

$$
F^{\prime}(r)=\log \left[P\left(\left\{K_{i j}\right\} \mid r, \hat{I}(\rho)\right)\right] \approx \ln \left[P\left(\left\{\lambda_{i j}\right\} \mid r,\left\{\widehat{\lambda}_{i j}(r)\right\}\right)\right]
$$

By using this approximation, the probability given in Eq. (11) can be interpreted as the probability of receiving $\lambda_{i j}\left(r_{0}\right)$ given the source intensity distribution $\left\{\hat{\lambda}_{i j}(\rho)\right\}$. Since it is assumed that $I_{0}(\rho)$ differs from $\hat{I}(\rho)$ by a zero-mean Gaussian process $I_{1}(\rho)$, it follows that the probability in Eq. (14) can be written as

$$
\begin{aligned}
P\left(\left\{\lambda_{i j}\right\} \mid r, \hat{I}(\rho)\right)= & \frac{1}{\sqrt{(2 \pi)^{M}\left|\sigma_{i j, \ell m}\right|}} \\
& \cdot \exp \left[-\frac{1}{2} \sum_{i j, \ell m}\left(\lambda_{i j}\right.\right. \\
& \left.\left.-\hat{\lambda}_{i j}(r)\right) \sigma_{i j, \ell m}^{-1}\left(\lambda_{\ell m}-\hat{\lambda}_{\ell m}(r)\right)\right]
\end{aligned}
$$

where $M$ is the total number of pixels used in the decision, and $\sigma_{i j, \ell m}^{-1}$ and $\left|\sigma_{i j, \ell m}\right|$ denote the matrix inverse and the determinant of the covariance matrix $\sigma_{i j, \ell m}$, respectively. The elements of the covariance matrix can be given by

$$
\begin{aligned}
\sigma_{i j, \ell m}(r) & =\left\langle\left(\hat{\lambda}_{i j}(r)-\lambda_{i j}(r)\right)\left(\hat{\lambda}_{\ell m}(r)-\lambda_{\ell m}(r)\right)\right\rangle \\
& =\int_{A_{i j}} \int_{A_{\ell m}} \phi\left(\rho-r, \rho^{\prime}-r\right) d^{2} \rho d^{2} \rho^{\prime}
\end{aligned}
$$

By differentiating the lıkelihood function in Eq. (14) and taking the expectation values, the Cramer-Rao bound of the estimator error variance can be given in terms of Eq. (12) (see Appendix) where

$$
\begin{aligned}
E\left[\left(\frac{\partial F^{\prime}(r)}{\partial x}\right)^{2}\right]= & {\left[\sum _ { i j , \ell m } \left(\left(\frac{\partial \hat{\lambda}_{i j}}{\partial x}\right)\left(\frac{\partial \widehat{\lambda}_{\ell m}}{\partial x}\right)\left[\sigma^{-1}\right]_{i j, \ell m}\right.\right.} \\
& \left.\left.+\left(\frac{\partial \sigma_{i j, \ell m}}{\partial x}\right)\left(\frac{\partial\left[\sigma^{-1}\right]_{i j, \ell m}}{\partial x}\right)\right)\right] \\
E\left[\left(\frac{\partial F^{\prime}(r)}{\partial y}\right)^{2}\right]= & {\left[\sum _ { i j , \ell m } \left(\left(\frac{\partial \hat{\lambda}_{i j}}{\partial y}\right)\left(\frac{\partial \widehat{\lambda}_{\ell m}}{\partial y}\right)\left[\sigma^{-1}\right]_{i j, \ell m}\right.\right.} \\
& \left.\left.+\left(\frac{\partial \sigma_{i j, \ell m}}{\partial y}\right)\left(\frac{\partial\left[\sigma^{-1}\right]_{i j, \ell m}}{\partial y}\right)\right)\right] \\
E\left[\left(\frac{\partial F^{\prime}(r)}{\partial x}\right)\left(\frac{\partial F^{\prime}(r)}{\partial y}\right)\right]= & {\left[\sum _ { i j , \ell m } \left(\left(\frac{\partial \hat{\lambda}_{i j}}{\partial x}\right)\left(\frac{\partial \hat{\lambda}_{\ell m}}{\partial y}\right)\left[\sigma^{-1}\right]_{i j, \ell m}\right.\right.} \\
& \left.\left.\left.+\left(\frac{\partial \sigma_{i j, \ell m}}{\partial x}\right)\left(\frac{\partial\left[\sigma^{-1}\right]_{i j, \ell m}}{\partial y}\right)\right)\right](17 c)\right]
\end{aligned}
$$

Equations (12) and (17) together present an analytical form of the mean square estimator error. Given the estimated intensity pattern $\hat{I}(\rho)$, and the intensity correlation function $\phi\left(\rho, \rho^{\prime}\right)$, the lower bound for the variance in estimating the image location can be calculated. Unfortunately, for general distributions of $\hat{I}(\rho)$ and $\phi\left(\rho, \rho^{\prime}\right)$, the expressions in Eqs. $(17 \mathrm{a}-\mathrm{c})$ are very difficult to evaluate. In order to obtain some insight into the functional dependence of tracking error variance and the source intensity error, some simplifications are required. In the following we shall present several simple cases that will illustrate these dependencies.

Example 1: White intensity noise with spectral density $\gamma^{2}$. That is,

$$
\left\langle I_{1}(\rho) I_{1}\left(\rho^{\prime}\right)\right\rangle=\frac{\gamma^{2}}{A} \delta\left(\rho-\rho^{\prime}\right)
$$

where $A$ is the area of a pixel element, and $\delta(x)$ is the Dirac delta function. An example of this type of intensity uncertainty is the random dark counts from the tracking detector. By using Eq. (16), the correlation matrix can be calculated. The result is

$$
\sigma_{i j, \ell m}=\gamma^{2} \delta_{i \ell} \delta_{j m}
$$


where $\delta_{i j}$ is the Kronecker delta. By substituting Eq. (20) into Eqs. $(17 \mathrm{a}-\mathrm{c})$, and using the fact that the $\sigma_{i j, \ell m}$ variables do not depend on $r$, the CRLB can be reduced to

$\operatorname{Var}\left(\left|\hat{r}-r_{0}\right|\right) \geqslant$

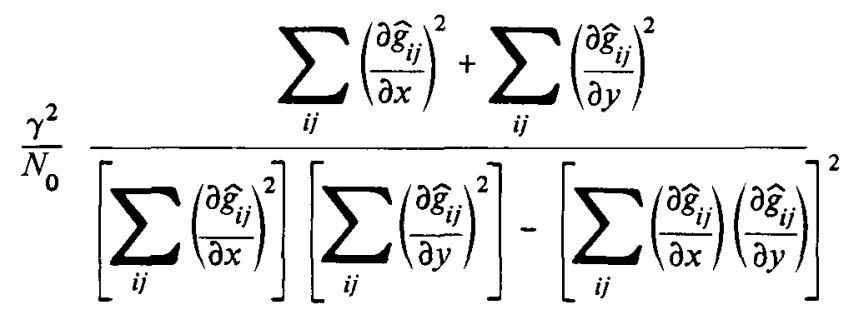

where the total detector SNR, $N_{0}$, is factored out by making the substitution $\lambda_{i j}(r)=N_{0} g_{i j}(r)$. It is easily seen from Eq. (20) that the variance in estimating the image location is directly proportional to the uncertainties in source intensity distribution $\gamma^{2}$. Furthermore, the variance of the estimator error decreases with increasing $N_{0}$ and, at a very high signal count, the variance is negligible.

Example 2: White intensity fluctuation with spectral density that is proportional to the total signal intensity. In other words,

$$
\phi\left(\rho, \rho^{\prime}\right)=\frac{\gamma^{2} N_{0}^{2}}{A} \delta\left(\rho-\rho^{\prime}\right)
$$

In this case the uncertainty in image brightness is proportional to the intensity of the image. An example of this type of intensity uncertainty is the unknown albedo variation of the source. An increase in the integration time at the tracking detector will only result in a proportional increase of the uncertainty. Under this condition, the CRLB reduces to

$$
\operatorname{Var}\left(\left|\widehat{r}-r_{0}\right|\right) \geqslant
$$

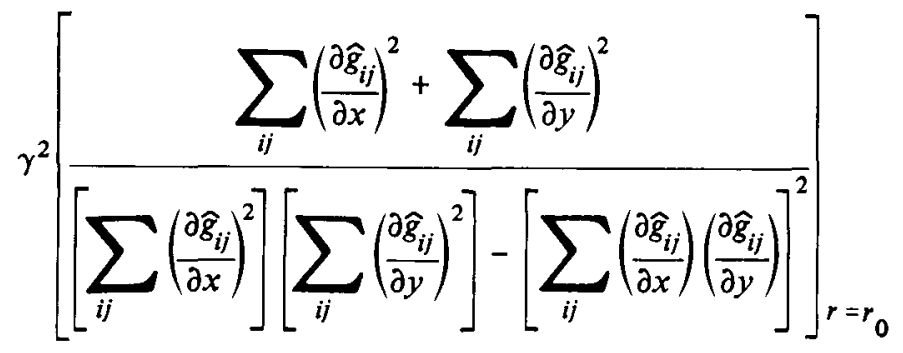

Note that even though the CRLB still depends linearly on $\gamma^{2}$, the lower bound no longer depends on the total detector SNR. Consequently, Eq. (22) represents an irreducible error floor for the estimator. For the simple test patterns shown in Fig. 1, the magnitude of the CRLB can be easily calculated to be $\gamma^{2}(b+2 a)^{3} / a$ and $\gamma^{2} \pi^{2} a^{2} / 2$, respectively.

\section{Tracking System Using Sun-Lit Earth as a Pointing Reference}

In a typical spatial tracking subsystem, the transmitter pointing information is derived from the image location of the reference source. The reference source can be either an uplink beacon laser, or the sun-lit Earth. The reference signal is received by the telescope and, after spatial and frequency filtering to cut down the background noise, is focused onto the tracking detector. The tracking detector is usually a focalplane array which spans the field-of-view (FOV) of the receiving optics, and can be implemented using a large-format CCD.

Deriving the angular coordinate of the reference source is equivalent to determining the position of its image. Since objects with angular separation less than the resolution limits cannot be resolved by the receiving optics, it is generally desirable to design the optics such that the pixel size on the focal plane array corresponds to the resolution limit of the telescope. Such a design provides maximum spatial information with a minimum number of pixels. For a spacecraft at a distance of $2.5 \mathrm{AU}$ using a $10 \mathrm{-cm}$ transmitter, the image of the Earth will span roughly 4-5 pixels. The required pointing accuracy, on the other hand, is less than $1 / 10$ of the transmitted beamwidth. Since the angular resolution of the telescope is equivalent to the minimal divergence of the transmitted optical signal, deriving the desired pointing accuracy of $1 / 10$ the beamwidth is therefore equivalent to locating the position of the receiving station to within $1 / 10$ of a pixel size based on the Earth image on the focal plane array.

When the intensity distribution of the pointing reference is known, as would be the case when an uplink beacon is used, the performance of the receiver is given by Eq. (6). The expected detector SNR, $N_{0}$, can be calculated using simple link analysis. By using the additional assumption that atmospheric scattering limits the angular divergence of the uplink beacon to about $20 \mu \mathrm{rad}$, the detector SNR can be approximated by

$$
N_{0} \approx 5 \times 10^{2} P_{s} D_{R}^{2} T / z^{2}
$$

where $P_{s}$ is the beacon power in watts, $D_{R}$ is the receiver diameter in meters, $T$ is the integration time, and $z$ is the link distance in AUs. Equation (23) was derived by assuming a 532-nm uplink beacon, and that the losses in optics and detector are 
negligible. The actual signal count can be much lower than that given by Eq. (23) due to these losses.

It is easily seen from Eq. (23) that, for a tracking detector operating at $1 \mathrm{KHz}$ using a $10-\mathrm{cm}$ diameter receiver at $1 \mathrm{AU}$, the required signal power for a $20-\mathrm{dB}$ SNR is $20 \mathrm{KW}$ ! Obviously, such a high power can be very costly to achieve. And if higher SNR is desired, the required beacon power can be even higher.

An alternative is to use the sun-lit Earth as a pointing reference. Sunlight reflection off the Earth can provide a large amount of signal power at the tracking detector. In fact, a simple calculation shows that a detector SNR higher than $10^{5}$ can be easily achieved. As a result, tracking error due to Poissoncount statistics is negligible. Unfortunately, the albedo of the Earth cannot be specified precisely. Cloud-cover can alter the surface reflectivity significantly, and a snow-covered surface can reflect up to one order of magnitude more sunlight than an exposed terrain. To further complicate the problem, these conditions are changing in time so that it is almost impossible to derive an accurate estimate of the Earth's albedo. Since the uncertainty is inherent to the brightness of the source, increasing the integration will only result in corresponding increases in that uncertainty. Consequently, there will be an irreducible error floor on the tracking system performance.

The actual impact of albedo uncertainty on the tracking system performance depends, of course, on the magnitude of the uncertainty and its spatial correlation. In general, the CRLB given by Eqs. (12) and (17) is very difficult to compute. For the simple test patterns shown in Fig. 1, a minimal standard deviation of 0.1 pixel requires that $\gamma$ be less than $0.1 \sqrt{a /(b+2 a)^{3}}$ and $0.045 / a$, respectively. If these examples are representative, then we will need to know the intensity to within 10 percent of the true value in order to limit the pointing error to within 0.1 pixel. Since the average Earth albedo variation is much more than 10 percent, a simple ML estimator cannot be expected to achieve the desired tracking accuracy.

\section{Conclusions}

Because of the large link distance involved, it is desirable that the optical communication package aboard a planetary spacecraft derive its pointing reference directly from the image of the sun-lit Earth on the tracking detector. Given the detector photocounts and the prior knowledge of the image intensity distribution, the maximum-likelihood spatial acquisition algorithm can be derived. The performance of the maximum-likelihood algorithm was analyzed by calculating the Cramer-Rao bound on the variance of acquisition error. It is shown that, when the intensity distribution of the pointing reference can be precisely characterized, the variance in estimating the receiver angular location decreases with increasing image intensity or detector exposure time. On the other hand, when the intensity distribution is not known in sufficient detail, the spatial tracking error variance will not decrease indefinitely with increasing exposure time. For a planetary spacecraft using the Sun-lit Earth as a pointing reference, the Earth's albedo cannot be precisely specified because of changing weather and ground conditions. Consequently, the $M L$ algorithm which derives the pointing reference based on the detector photocounts cannot be expected to provide an accurate pointing reference.

It should be noted, however, that the results presented in this study were derived by assuming that the receiver derives its pointing reference based on a single frame of the image. In other words, the receiver estimates the image location, $r$, based on the receiver count statistics and an assumed source intensity distribution, $\widehat{I}(\rho)$. When multiple images of the pointing reference are available, the receiver can then jointly estimate the true source distribution, $I_{0}(\rho)$, and the image location, $r$. For such a receiver, the tracking system performance will not be limited by the intensity uncertainty. In fact, with a sufficiently large number of look angles, one could derive an estimate $\widehat{I}(\rho)$ which closely approximates $I_{0}(\rho)$ and, consequently, the variance in estimating $r$ could be reduced to an acceptable level.

\section{References}

[1] K. Winick, "Cramer-Rao Lower Bound on the Performance of Charge-Coupled-Device Optical Position Estimators," J. Opt. Soc. Am. A, vol. 3, no. 11, pp. 1809-1815, November 1986.

[2] M. Win, "Acquisition and Tracking for Optical Communications," submitted to OE-LASE'89, Los Angeles, California, January 1989.

[3] H. Van Tree, Detection, Estimation, and Modulation Theory, New York: Wiley, 1968. 
(a)

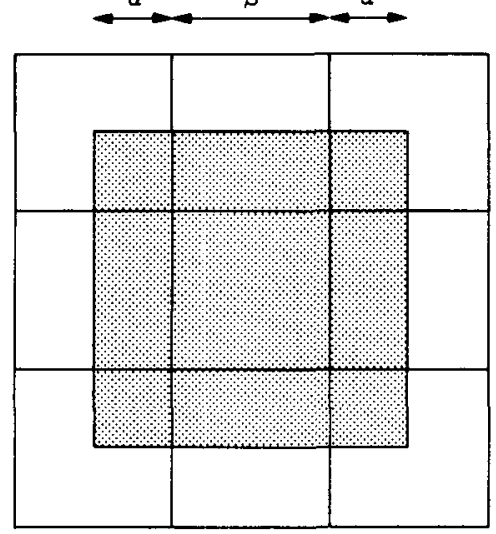

$G\left(r_{0}\right)=\alpha(\beta+2 \alpha)$ (b)

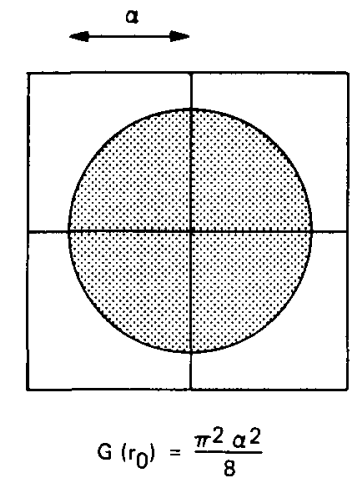

Fig. 1. The value of $G\left(r_{0}\right)$ for two simple examples of source intensity distributions over the detector focal plane. The solid grids represent the CCD pixels, and the shaded areas represent the image of the pointing reference. The image intensities are assumed to be uniformly distributed. 


\section{Appendix}

\section{Derivation of Equation (16)}

The logarithm of the joint probability distribution $P\left(\left\{\lambda_{i j}\right\} \mid r, I(\rho)\right)$, is

$$
\ln \left[P\left(\left\{\lambda_{i j}\right\} \mid r, \hat{I}(\rho)\right)\right]=-\frac{1}{2} \ln \left|\sigma_{i j, \ell m}\right|-\frac{1}{2} \sum_{i j, \ell m}\left(\lambda_{i j}-\hat{\lambda}_{i j}(r)\right)\left[\sigma^{-1}\right]_{i j, \ell m}\left(\lambda_{\ell m}-\hat{\lambda}_{\ell m}\right)
$$

By expanding $E\left[\left(\partial \ln \left(P\left(\left\{\lambda_{i j}\right\} \mid r, I(\rho)\right) / \partial x\right)^{2}\right]\right.$ into an integral form, and using the fact that

$$
\int\left(\partial^{2} P\left(\left\{\lambda_{i j}\right\}\right) / \partial x^{2}\right) d\left\{\lambda_{i j}\right\}=0
$$

it follows that

$$
\begin{aligned}
E\left[\left(\frac{\partial \ln \left[P\left(\left\{\lambda_{i j}\right\} \mid r, I(\rho)\right)\right]}{\partial x}\right)^{2}\right] & =\int\left(\frac{\partial P\left(\left\{\lambda_{i j}\right\}\right) / \partial x}{P\left(\left\{\lambda_{i j}\right\}\right)}\right)^{2} P\left(\left\{\lambda_{i j}\right\}\right) d\left\{\lambda_{i j}\right\} \\
& =\int\left\{\left(\frac{\partial P\left(\left\{\lambda_{i j}\right\}\right) / \partial x}{P\left(\left\{\lambda_{i j}\right\}\right)}\right)^{2}+\left(\frac{\partial^{2} P\left(\left\{\lambda_{i j}\right\}\right) / \partial x^{2}}{P\left(\left\{\lambda_{i j}\right\}\right)}\right)\right\} P\left(\left\{\lambda_{i j}\right\}\right) d\left\{\lambda_{i j}\right\} \\
& =\int \frac{\partial^{2} \ln \left[P\left(\left\{\lambda_{i j}\right\} \mid r, I(\rho)\right)\right]}{\partial x^{2}} P\left(\left\{\lambda_{i j}\right\}\right) d\left\{\lambda_{i j}\right\} \\
& =-E\left[\frac{\partial^{2} \ln \left[P\left(\left\{\lambda_{i j}\right\} \mid r, I(\rho)\right)\right]}{\partial x^{2}}\right]
\end{aligned}
$$

By differentiating Eq. (A-1) and using the fact that $E\left[\partial P\left(\lambda_{i j} \mid r, \hat{I}(\rho)\right) / \partial x\right]$ is equal to zero for all $r$, one can show that

$$
\begin{aligned}
\frac{\partial}{\partial x} E\left[\frac{\partial}{\partial x} P\left(\left\{\lambda_{i j}\right\} \mid r, \widehat{I}(\rho)\right)\right] & =-\frac{\partial^{2}}{\partial x} \ln \left|\sigma_{i j, \ell m}\right|-\frac{1}{2} \sum \sigma_{i j, \ell m} \frac{\partial^{2}\left[\sigma^{-1}\right]_{i j, \ell m}}{\partial x^{2}}-\frac{1}{2} \sum \sigma_{i j, \ell m} \frac{\partial \sigma_{i j, \ell m}}{\partial x} \frac{\partial\left[\sigma^{-1}\right]_{i j, \ell m}}{\partial x} \\
& \equiv 0
\end{aligned}
$$

In deriving Eq. (A-3), we have used the assumption that $\left\{\lambda_{i j}\right\}$ are Gaussian distributed with mean $\left\{\hat{\lambda}_{i j}\right\}$.

By differentiating Eq. (A-1) and taking the expectation values of $\lambda_{i j}$, Eq. (A-2) is reduced to

$E\left[\left(\frac{\partial}{\partial x} \ln \left[P\left(\left\{\lambda_{i j}\right\} \mid r, \hat{I}(\rho)\right)\right]\right)^{2}\right]=\frac{1}{2} \frac{\partial^{2}}{\partial x^{2}} \ln \left|\sigma_{i j, \ell m}\right|+\frac{1}{2} \sum_{i j, \ell m} \sigma_{i j, \ell m} \frac{\partial^{2}\left[\sigma^{-1}\right]_{i j, \ell m}}{\partial x^{2}}+\frac{1}{2} \sum_{i j, \ell m}\left(\frac{\partial \hat{\lambda}_{i j}}{\partial x}\right)\left[\sigma^{-1}\right]_{i j, \ell m}\left(\frac{\partial \hat{\lambda}_{\ell m}}{\partial x}\right)$ 
By substituting Eq. (A-3) into (A-4), it is seen that

$$
E\left[\left(\frac{\partial}{\partial x} \ln \left[P\left(\left\{\lambda_{i j}\left(r_{0}\right)\right\} \mid r, \widehat{I}(\rho)\right)\right]\right)^{2}\right]=\left[\sum_{i j, \ell m}\left(\left(\frac{\partial \lambda_{i j}}{\partial x}\right)\left(\frac{\partial \lambda_{\ell m}}{\partial x}\right)\left[\sigma^{-1}\right]_{i j, \ell m}+\left(\frac{\partial \sigma_{i j, \ell m}}{\partial x}\right)\left(\frac{\partial\left[\sigma^{-1}\right]_{i j, \ell m}}{\partial x}\right)\right)\right]
$$

Similarly, it can be shown that

$$
E\left[\left(\frac{\partial}{\partial y} \ln \left[P\left(\left\{\lambda_{i j}\left(r_{0}\right)\right\} \mid r, \hat{I}(\rho)\right)\right]\right)^{2}\right]=\left[\sum_{i j, \ell m}\left(\left(\frac{\partial \lambda_{i j}}{\partial y}\right)\left(\frac{\partial \lambda_{\ell m}}{\partial y}\right)\left[\sigma^{-1}\right\}_{i j, \ell m}+\left(\frac{\partial \sigma_{i j, \ell m}}{\partial y}\right)\left(\frac{\partial\left[\sigma^{-1}\right]_{i j, \ell m}}{\partial y}\right)\right)\right]
$$

and

$$
E\left[\left(\frac{\partial \ln \left[P\left(\left\{\hat{\lambda}_{i j}\left(r_{0}\right)\right\} \mid r, \hat{I}(\rho)\right)\right]}{\partial x}\right)\left(\frac{\partial \ln \left[P\left(\left\{\hat{\lambda}_{i j}\left(r_{0}\right)\right\} \mid r, \widehat{I}(\rho)\right)\right]}{\partial y}\right)\right]=\left[\sum_{i j, \ell m}\left(\left(\frac{\partial \lambda_{i j}}{\partial x}\right)\left(\frac{\partial \lambda_{\ell m}}{\partial y}\right)\left[\sigma^{-1}\right]_{i j, \ell m}+\left(\frac{\partial \sigma_{i j, \ell m}}{\partial x}\right)\left(\frac{\partial\left[\sigma^{-1}\right]_{i j, \ell m}}{\partial y}\right)\right)\right]
$$

